\title{
Low Complexity Quasi-Optimal Detector for Generalized Spatial Modulation
}

\author{
Majed Saad, Hussein Hijazi, Ali Chamas Al Ghouwayel, Faouzi Bader and Jacques Palicot
}

\begin{abstract}
Generalized Spatial Modulation (GSM) is a promising technique for high data-rates systems where the complexity of the optimal joint detector based on the Maximum Likelihood (ML) criterion increases dramatically in terms of the modulation order and the number of activated transmit antennas. In this paper, a novel detection scheme for GSM systems denoted by $\mathrm{O}^{2} \mathrm{SIC}-\mathrm{ML}$ is proposed to achieve the optimal performance with a controllable complexity-performance trade-off. The proposed detector is accompanied with ordering techniques for the transmit antenna combination and symbols to further reduce the complexity and improve the performance, respectively. The result reveals that $\mathrm{O}^{2}$ SIC-ML allows reaching the non-linear ML detector performance with a $99 \%$ complexity reduction.
\end{abstract}

Index Terms-Multiple-Input Multiple-Output (MIMO), Generalized Spatial Modulation (GSM), Detectors, Equalizers, Maximum Likelihood (ML) detection, Sphere Decoding (SD), Ordered Successive Interference Cancellation (OSIC), Complexity.

\section{INTRODUCTION}

G ENERALIZED Spatial Modulation (GSM) [1] is a spectral and energy-efficient scheme in the spatial Index Modulation (IM) domain. More precisely, GSM scheme conveys information bits in the signal domain by the transmitted symbols and in the spatial domain by the index of activated Transmit Antenna Combination (TAC). This Multiple-InputMultiple-Output (MIMO) system generalizes the spatial multiplexing (SMX) and many other spatial IM schemes.

However, the optimal joint Maximum Likelihood (ML) that detects the activated TAC and the transmitted symbols suffers from high complexity, especially when this scheme is used with large modulation order and number of activated Transmit Antennas (TAs) to reach high rates. Consequently, a low complexity linear detection is proposed in [1], but it leads to substantial performance degradation. Another low complexity detector named Ordered Block Minimum Mean-Squared Error (OB-MMSE) and its improved version have been proposed in [2], [3]. The OB-MMSE based detector permits to enhance the performance without reaching the optimal performance unless high receive diversity is set: $N_{r} \geq 2 N_{a}$ where $N_{r}$ and $N_{a}$ are the number of receive and activated TAs, respectively. More powerful detection based on Sphere Decoding (SD) was also

M. Saad and J. Palicot are with Signal, Communication \& Embedded Electronics (SCEE) research group, CentraleSupéleclIETR-Campus of Rennes, Cesson-Sévigné, 35510 Brittany, France e-mail: majed.saad@ieee.org, \{jacques.palicot\}@supelec.fr.

H. Hijazi is with CCE department, Lebanese International University, Beirut, Lebanon email: hussein.hijazi@liu.edu.lb.

A. Chamas Al Ghouwayel is with the School of Engineering EFREI, 94800 Villejuif-Paris, France email: ali.ghouwayel@efrei.fr

Faouzi Bader is with Institut Supérieur d'Electronique de Paris, 75006 Paris, France email: faouzi.bader@isep.fr. explored, but its complexity is affected by the initial search radius [4]. Hence, two SD approaches for spatial modulation, Transmit-based SD (Tx-SD) and Receive-based SD (Rx-SD), are developed in [4] in order to reduce the conventional SD complexity. Recently, two improved SD techniques based on Tree search-SD (T-SD) and Path search-SD (P-SD) were proposed to provide greater complexity reduction [5], which is still much higher than linear detectors.

In this paper, a novel detection technique for GSM is proposed and denoted by Ordered TAC- Ordered Successive Interference Cancellation with $\mathrm{ML}$ verification $\left(\mathrm{O}^{2} \mathrm{SIC}-\mathrm{ML}\right)$. This technique is inspired by [6], [7] that provides a balanced performance-complexity trade-off for MIMO SMX systems. In contrast to [6], [7], where only Amplitude-Phase Modulation (APM) symbols are detected, $\mathrm{O}^{2}$ SIC-ML can estimate the real bits encapsulated by the APM symbols and/or the virtual bits conveyed by the index of the activated TAC, so $\mathrm{O}^{2}$ SIC-ML can be adapted to MIMO SMX and all other GSM special cases. The proposed detector aims to reach a quasi-optimal performance with low complexity and provide a controllable detector to achieve a balanced complexity-performance tradeoff. In order to mitigate the inter-antenna interference induced by the simultaneous APM transmission from multiple activated TAs, this proposed detector applies the conventional OSIC method [8] for each TAC while considering different potential candidates (neighbor symbols) instead of a single symbol. Afterward, an ML verification is applied on this reduced set of candidates to enhance the performance. Moreover, in order to maintain a low complexity, a TAC ordering layer is incorporated with an early termination.

The rest of the paper is organized as follows. The GSM system model is shown in Section II. In section III, the GSM $\mathrm{O}^{2} \mathrm{SIC}-\mathrm{ML}$ detector algorithm is explained and analyzed in terms of computational complexity. Section IV discusses the results of the proposed method with respect to linear and nonlinear detectors. Finally, Section V concludes the paper.

The following notations are adopted in the paper. Boldface with uppercase/lowercase letters $\mathbf{X} / \mathbf{x}$ are used for matrices and vectors respectively. (. $)^{H},(.)^{\dagger}$ and $(.)^{-1}$ are used to denote respectively the Hermitian, the pseudo-inverse and the inverse of a matrix. $C \mathcal{N}\left(\mu, \sigma^{2}\right)$ denotes the complex normal distribution of a random variable having mean $\mu$ and variance $\sigma^{2}$. $\|$.$\| stands for the Frobenius norm.$

\section{SySTEM MODEL}

Consider a GSM system with $N_{t}$ TAs and $N_{r}$ receive antennas, where $N_{a}$ TAs are only activated to convey additional bits by the TAC index as depicted in Fig. 1. These activated TAs can transmit the same $M$-ary APM symbol for diversity gain 


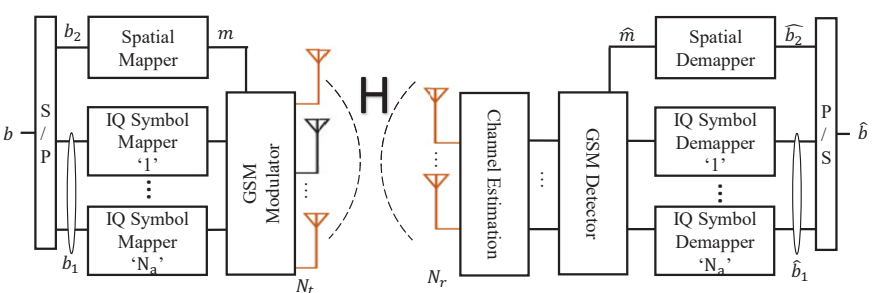

Fig. 1: GSM transceiver System Model with $N_{a}$ activated transmit antennas from $N_{t}$, and $N_{r}$ receive antennas.

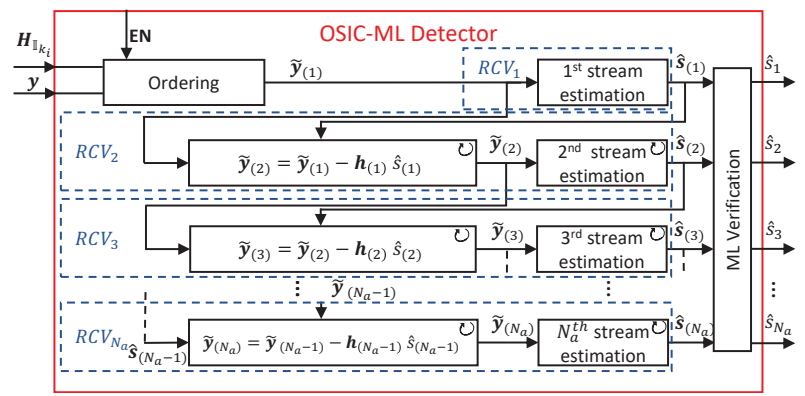

Fig. 2: OSIC-ML detector model with linear stream estimators. EN is a boolean variable used to activate the ordering technique prior to APM detection and interference cancellation.

or different APM symbols for multiplexing gain. The possible number of legitimate TAC $N=2^{\left\lfloor\log _{2}\left(C_{N_{a}}^{N_{t}}\right)\right\rfloor}$, and the number of conveyed bits per GSM symbol is $N_{a} \log _{2} M+\log _{2} N$ when multiplexing is considered. The received signal vector $\mathbf{y}$ is given by:

$$
\mathbf{y}=\mathbf{H x}+\mathbf{v},
$$

where $\mathbf{H}=\left[\mathbf{h}_{1}, \ldots, \mathbf{h}_{N_{t}}\right]$ is the $N_{r} \times N_{t}$ MIMO channel matrix with $\mathbf{h}_{k}$ is the column vector of $N_{r}$ elements, $\mathbf{x}=$ $\left[x_{1}, \ldots, x_{N_{t}}\right]^{T}$ is the transmitted vector that contains only $N_{a}$ APM symbols $\left(s_{1}, s_{2} \ldots s_{N_{a}}\right)$ on the activated TAs according to selected TAC, and $\mathbf{v}$ is $N_{r} \times 1$ noise vector where its elements obeys the independent and identically distributed (i.i.d.) additive white Gaussian noise with zero-mean and variance of $\sigma_{v}^{2}$, i.e, $\operatorname{CN}\left(0, \sigma_{v}^{2}\right)$.

At the receiver side, the detection of the $N_{a}$ APM symbols and the activated TAC can be jointly estimated using ML detector as follows:

$$
\left(\widehat{\mathbf{s}}_{\mathrm{ML}}, \hat{\mathbb{I}}\right)=\underset{\mathbf{s} \in \chi, \mathbb{I} \in I}{\arg \min }\left\|\mathbf{y}-\tilde{\mathbf{H}}_{\mathbb{I}} \mathbf{S}\right\|^{2}
$$

where $\chi$ contains the vectors of all possible APM symbols combinations $\left(M^{N_{a}}\right), \mathcal{I}=\left\{\mathbb{I}_{1}, \mathbb{I}_{2}, \ldots, \mathbb{I}_{N}\right\}$ is the set of all legitimate TACs where each $\mathbb{I}_{i}$ contains the indices of activated TAs, and $\tilde{\mathbf{H}}_{\mathbb{I}}$ is the sub-matrix of $\tilde{\mathbf{H}}$ with $N_{a}$ columns corresponding to activated TAs in $\mathbb{I}$. In the sequel, the estimated channel $\tilde{\mathbf{H}}$ is assumed perfectly known at the receiver.

\section{Proposed GSM O² SIC-ML Detector \\ A. Algorithm}

In this section, we propose the $\mathrm{O}^{2}$ SIC-ML detector for GSM systems that considers the interference cancellation similar to OSIC detector. However, it will be performed on several possible candidate symbols to reach the near-optimal performance with a good complexity-performance trade-off. Firstly, this detector will sort the $N$ possible legitimate TACs to start the algorithm on the most probable activated TAC. For this reason, a metric $z_{p}$ for each transmitted antenna is computed to equalize the channel with respect to the potential transmitted symbol at the $p^{t h}$ TA. The weights $w_{i}$ of each

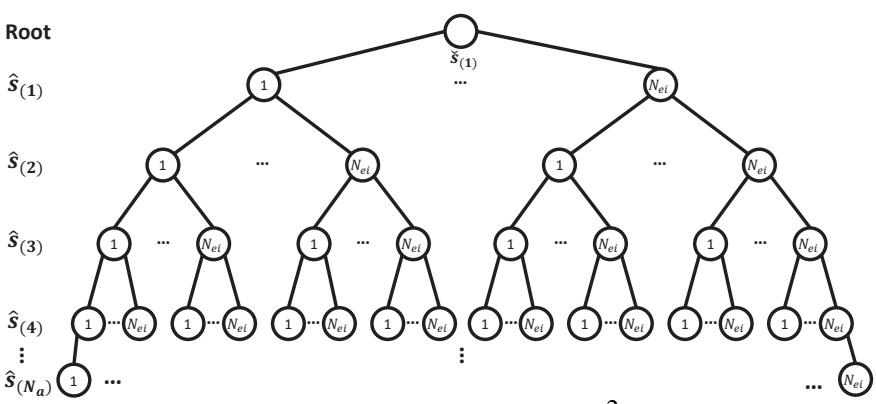

Fig. 3: Tree search for the OSIC-ML stage of $\mathrm{O}^{2}$ SIC-ML with height $N_{a}$ and branching factor $N_{e i}$. The corresponding branches of each node in the tree are numbered from 1 to $N_{e i}$.

TAC $i$ are extracted based on the different combinations of $N_{a} z_{p}$ metrics as shown below:

$$
\begin{aligned}
& z_{p}=\mathbf{h}_{p}^{\dagger} \mathbf{y} \text { with } \mathbf{h}_{p}^{\dagger}=\frac{\mathbf{h}_{p}^{H}}{\mathbf{h}_{p}^{H} \mathbf{h}_{p}} \\
& w_{i}=\left|z_{i_{1}}\right|^{2}+\left|z_{i_{2}}\right|^{2}+\ldots+\left|z_{i_{N a}}\right|^{2}=\sum_{n=1}^{N_{a}}\left|z_{i_{n}}\right|^{2},
\end{aligned}
$$

where $p \in\left\{1,2, \ldots, N_{t}\right\}, i \in\{1,2, \ldots, N\}$ and $i_{n}$ is the index of the $n^{\text {th }}$ activated TA in the $i^{\text {th }}$ TAC. Then, these weights $\mathbf{w}=\left[w_{1}, \ldots, w_{N}\right]$ are sorted in descending order:

$$
\left[k_{1}, k_{2}, \ldots, k_{N}\right]=\arg \operatorname{sort}(\mathbf{w}, \text { descending }),
$$

where $k_{1}$ is the index of the TAC with the maximum weight. Then, an OSIC-ML is performed for each TAC $\mathbb{I}_{k_{i}}$ following the ordered list in (5) using the submatrix $\mathbf{H}_{\mathbb{I}_{k_{i}}}$ in order to detect the $N_{a}$ APM symbols $\hat{\mathbf{s}}=\left[\hat{s}_{1}, \hat{s}_{2}, \ldots, \hat{s}_{N_{a}}\right]$, as shown in Fig. 2. The final decision is deduced by taking the TAC and APM symbol vector that gives the overall minimum distance.

More precisely, the OSIC-ML receiver shown in Fig. 2 is performed for each TAC $\mathbb{I}_{k_{i}}$, where any symbol ordering technique with MIMO SMX for better interference cancellation can be adopted. In the following, the detection without any symbol ordering and with the simplest symbol ordering (without/with power priority) based on the channel matrix columns norm $\left(\left\|\mathbf{h}_{p}\right\|^{2}\right)$ is considered. Note that these $N_{t}$ norms are calculated once and sorted in decreasing order to identify the order of symbol detection for each considered TAC. The notation $s_{(n)}$ and $s_{n}$ represent the $n^{\text {th }}$ APM symbol after ordering and the transmitted symbol from the $n^{\text {th }}$ activated TA, respectively, similar notation is used for the corresponding h. Thus, the received signal $\mathbf{y}$ can be rewritten as:

$$
\mathbf{y}=\mathbf{h}_{(1)} s_{(1)}+\mathbf{h}_{(2)} s_{(2)}+\mathbf{h}_{(3)} s_{(3)}+\ldots+\mathbf{h}_{\left(N_{a}\right)} s_{\left(N_{a}\right)}+\mathbf{v} .
$$

Hence, each of the $N_{a}$ Receive Circuits (RCV) shown in Fig. 2 (dotted box) will cancel the interference contribution of the previous $(n-1)$ detected APM symbol from the reducedinterference observation $\tilde{\mathbf{y}}_{(n-1)}$, then estimate the $n^{\text {th }}$ symbol using linear equalizer/detector (e.g. Zero-Forcing (ZF) or any other). The interference cancellation step can be expressed as:

$$
\tilde{\mathbf{y}}_{(n)}=\tilde{\mathbf{y}}_{(n-1)}-\mathbf{h}_{(n-1)} \hat{s}_{(n-1)} .
$$

It should be noted that $\tilde{\mathbf{y}}_{(1)}=\mathbf{y}$ so the first APM symbol is detected with complete interference. In the following, the $\mathrm{ZF}$ equalizer will be used, where its weight matrix $\mathbf{W}_{Z F}$ is the pseudo-inverse of the sub-matrix with the columns of $H_{\mathbb{I}_{k_{i}}}$ corresponding to non-detected symbols only. The linear 
equalizer gives the raw estimation $\breve{s}$ that will be quantized to the sliced estimation $\hat{s}$ before the interference cancellation. Upon this step, an OSIC detection is performed, but the misdetection of one of the first $N_{a}-1$ APM symbols leads to error propagation to subsequent RCVs, and it will highly impact the system performance. To overcome this drawback, we propose to consider not only the sliced $\hat{s}$ but the $N_{e i}$ sliced neighbors near the raw estimated symbol $\breve{s}$. Consequently, the next RCV will consider all these $N_{e i}$ neighbors, and so on until the last RCV circuit is reached. This process can be described by a tree search of height $N_{a}$ and branching factor $N_{e i}$ as depicted in Fig. 3, where $\hat{\mathbf{s}}_{(i)}$ contains the different sliced neighbors at each $i$-th activated antenna and the interference cancellation of (7) should be run over the different symbols of vector $\hat{\mathbf{s}}_{(i)}$. Thus, the number of possible $\mathbf{s}$ vectors provided by the tree for a given TAC is $N_{e i} N_{a}$, that are stored in the set C. Note that this detector becomes an ML when $N_{e i}=M$, but choosing a smaller $N_{e i}$ permits to avoid the tremendous complexity increase with large $M$-ary APM and $N_{t}$ TAs as it will be shown in the next sub-section. Consequently, this reduced set $\mathbf{C}$ is passed to the ML verification module to deduce $\hat{\mathbf{s}}_{\text {OSIC-ML }}$ for the vector $\mathbf{s}$ :

$$
\begin{aligned}
\widehat{\mathbf{s}}_{\text {OSIC-ML }} & =\underset{\hat{\mathbf{s}} \in \mathbf{C}}{\arg \min }\left\|\mathbf{y}-\mathbf{H}_{\mathbb{I}_{k_{j}}} \hat{\mathbf{s}}\right\|^{2} \\
& =\underset{\hat{\mathbf{s}} \in \mathbf{C}}{\arg \min }\left\|\mathbf{W}_{Z F} \mathbf{y}-\hat{\mathbf{s}}\right\|^{2} .
\end{aligned}
$$

The described $\mathrm{O}^{2}$ SIC-ML algorithm is performed successively for the ordered TACs until the computed distance in (8) satisfies the following condition:

$$
\left\|\mathbf{y}-\mathbf{H}_{\mathbb{I}_{k_{j}}} \hat{\mathbf{s}}\right\|^{2} \leq V_{t h},
$$

where $V_{t h}$ is a predefined threshold $V_{t h}=N_{r} \sigma_{v}^{2}$. This threshold is set for which the transmitted vector is correctly estimated, and thus the distance contains only the noise power. Note that this termination will occur in the early search stage with one of the TACs of highest weights, and thus it allows a further complexity reduction compared to the joint $\mathrm{ML}$ that searches in the $\left(N M^{N_{a}}\right)$ candidate vectors. Therefore, the proposed GSM O ${ }^{2}$ SIC-ML detector provides a good controlled performance-complexity trade-off by selecting a suitable $1 \leq N_{e i} \leq M$. The $\mathrm{O}^{2}$ SIC-ML algorithm is summarized in Algorithm 1 using the Matlab matrix notations. The main procedure deduces the final decisions $(\hat{\mathbb{I}}, \hat{s})$, while the function OSICML (line (18) to (25)) with the help of recursion function (line (26) to (39)) estimates $\widehat{\mathbf{s}}_{\text {OSICML for a }}$ given TAC. Note that $O($.$) denotes the quantization operation$ that gives the $N_{e i}$ nearest sliced neighbors of the raw estimate $\check{s}_{(i)}$, and the used 'min' operator in line (24) of Algorithm 1 returns the minimum distance and its argument. The input to this algorithm can be the ordered $\tilde{\mathbf{y}}$, and $\mathrm{ZF}$ equalizer can be replaced by the MMSE equalizer to deduce the raw estimations.

\section{B. Complexity Analysis}

The computational complexity of $\mathrm{O}^{2} \mathrm{SIC}-\mathrm{ML}$ is divided into the main algorithm complexity and the complexity of the ZF weight matrices pre-computation, where the latter is performed after each channel estimation (i.e., once per frame of $N_{s}$ symbols). These pre-computations include the $\mathrm{ZF}$ weight matrix estimation (line 20) and those for the raw estimations (line 33),

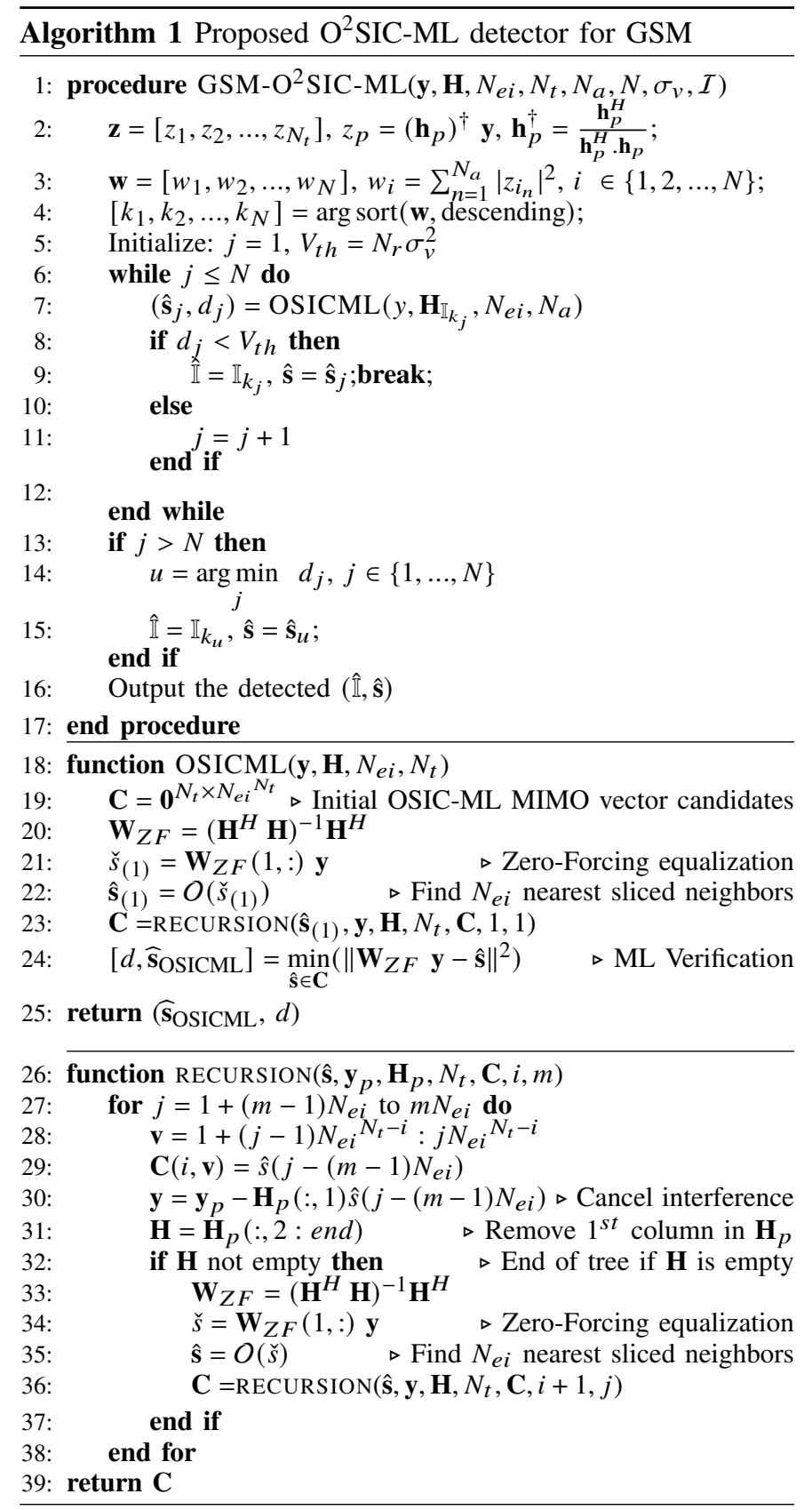

and their complexities in terms of Complex Multiplications (CMs) and Complex Additions (CAs) can be expressed as follows:

$$
\begin{aligned}
C_{\mathrm{pre}}^{C M} & =\sum_{i=0}^{N_{a}-1}\left(\left(N_{a}-i\right)^{3}+2\left(N_{a}-i\right)^{2} N_{r}\right) \\
C_{\mathrm{pre}}^{C A} & =\sum_{i=0}^{N_{a}-1}\left(\left(N_{a}-i\right)^{3}+\left(N_{a}-i\right)^{2}\left(N_{r}-1\right)\right. \\
& \left.+\left(N_{a}-i\right)\left(N_{a}-i-1\right) N_{r}\right)
\end{aligned}
$$

which is the sum of pseudo-inverse complexities for matrices of size $N_{\text {rem }} \times N_{r}$, where the number of remained un-detected APM symbols $N_{\text {rem }}$ decreases progressively from $N_{a}$ to 1 in the nested calls. Note that the number of Real Multiplications (RMs) and Real Additions (RAs) can be deduced by considering: $1 \mathrm{CM}$ contains $4 \mathrm{RMs}$ and $2 \mathrm{RAs}$, while $1 \mathrm{CA}$ includes 2 RAs.

The complexities of the $\mathrm{O}^{2}$ SIC-ML main functions in terms of RMs and RAs are summarized in Table I. Note that the raw estimation of APM symbols is a row multiplication of 
TABLE I: Computational complexity calculation for the main functions in the proposed detector.

\begin{tabular}{||c|c|l|l|l|l||}
\hline Function & TAC ordering & $\begin{array}{l}\text { Raw } \\
\text { estimation }\end{array}$ & $\begin{array}{l}\text { APM quantization } \\
\& \text { Neighbors search }\end{array}$ & $\begin{array}{l}\text { Interference } \\
\text { Cancellation }\end{array}$ & $\begin{array}{l}\text { ML } \\
\text { verification }\end{array}$ \\
\hline \hline RM/execution & $N_{t}\left(12 N_{r}+2\right)$ & $3 N_{r}$ & $2 M$ & $4 N_{r}$ & $2 N_{a}$ \\
\hline RA/execution & $N_{t}\left(10 N_{r}-3\right)+N\left(N_{a}-1\right)$ & $5 N_{r}-2$ & $3 M$ & $4 N_{r}$ & $4 N_{a}-1$ \\
\hline Number of executions & 1 & $\bar{N} \frac{N_{e i} N_{a}-1}{N_{e i}-1}$ & $\bar{N} \frac{N_{e i} N_{a}-1}{N_{e i}-1}$ & $\bar{N}\left(\frac{N_{e i} N_{a}-1}{N_{e i}-1}-1\right)$ & $\bar{N} N_{e i}^{N_{a}}$ \\
\hline
\end{tabular}

$\mathbf{W}_{Z F}$ by $\mathbf{y}$ (line (21) and (34) of Algorithm 1), and thus each $C M$ in these steps can be computed with 3 RMs and 3 RAs since $\mathbf{W}_{Z F}$ is known in advance [9, Eq. (12)]. The APM quantization and neighbor search in line (22) and (35) includes $M$ Euclidean Distances (EDs) at symbol level $\left(|\check{s}-s|^{2}\right)$ for each execution, while the ML verification for given TAC contains $N_{e i}{ }^{N_{a}}$ EDs calculation for vector of size $N_{a}$. Note that the EDs square is directly considered in the complexity to avoid redundant operations. The early termination with TAC ordering reduces the number of tested TAC to $\bar{N}$, where $\bar{N} \leq N$. Thus, the total computational complexity $C$ of the proposed detector in terms of the number of RMs, RAs and floating-point operations (flops) are given by:

$$
\begin{aligned}
C_{\mathrm{O}^{2} \mathrm{SIC}-\mathrm{ML}}^{R M}= & 4 \bar{N} \frac{C_{\mathrm{pre}}^{C M}}{N_{s}}+N_{t}\left(12 N_{r}+2\right) \\
& +\bar{N}\left(\left(7 N_{r}+2 M\right)\left(\frac{N_{e i}^{N a}-1}{N_{e i}-1}\right)-4 N_{r}+2 N_{a} N_{e i}^{N_{a}}\right) \\
C_{\mathrm{O}^{2} \mathrm{SIC}-\mathrm{ML}}^{R A}= & 2 \bar{N} \frac{C_{\mathrm{pre}}^{C M}+C_{\mathrm{pre}}^{C A}}{N_{s}}+N_{t}\left(10 N_{r}-3\right)+N\left(N_{a}-1\right) \\
& +\bar{N}\left(\left(9 N_{r}+2 M-2\right)\left(\frac{N_{e i}^{N_{a}}-1}{N_{e i}-1}\right)-4 N_{r}+\left(4 N_{a}-1\right) N_{e i}^{N_{a}}\right) \\
C_{\mathrm{O}^{2} \mathrm{SIC}-\mathrm{ML}}^{\text {flops }}= & C_{\mathrm{O}^{2} \mathrm{SIC}-\mathrm{ML}}^{R M}+C_{\mathrm{O}^{2} \mathrm{SIC}-\mathrm{ML}}^{R A}
\end{aligned}
$$

Note that the complexity of any pre-processing steps becomes negligible when a large frame length is used due to slow channel variation, and they have a more significant impact with fast channel variations.

The complexity of ML detector in terms of flops is expressed as:

$$
C_{\mathrm{ML}}=N M^{N_{a}}\left(8 N_{r} N_{a}+4 N_{r}-1\right)
$$

\section{Simulations RESUlTS AND DISCUSSIONS}

The GSM system's performance with the proposed $\mathrm{O}^{2} \mathrm{SIC}$ ML detector is compared to different existing linear and non-linear detectors (ML, Tx-SD, Rx-SD, Tree-SD, PathSD [11], ZF, OB-MMSE, OB-MMMSE-Reduced Complexity (OB-MMSE-RC)[16], L-first-MMSE [15], and Sorting Assisted Successive Sphere Decoding Algorithm (SA-SSDA) [17]). In addition, a Reduced complexity SD (Rc-SD) is considered by using the candidate sorting technique proposed in [10]. The average uncoded Bit Error Rate (BER) versus SNR is evaluated over different Rayleigh channel realizations by Monte Carlo simulations with $3 \times 10^{5}$ frames where each one contains 100 symbols. The proposed detector is considered with/without APM ordering.

Figure 4 shows the performance of different detectors with $N_{t}=N_{r}=5, N_{a}=[2,3], N_{e i}=2$, and $M=[16,32]$ QAM. As expected the performance of all nonlinear detectors, ML and SD, are overlapped. It is clear that $\mathrm{O}^{2}$ SIC-ML with $N_{e i}=2$ achieves the performance of optimal non-linear detectors when $N_{a}=2$, and a gap of $0.3(0.8) \mathrm{dB}$ appears when $N_{a}=3$ using the proposed detector with (without)

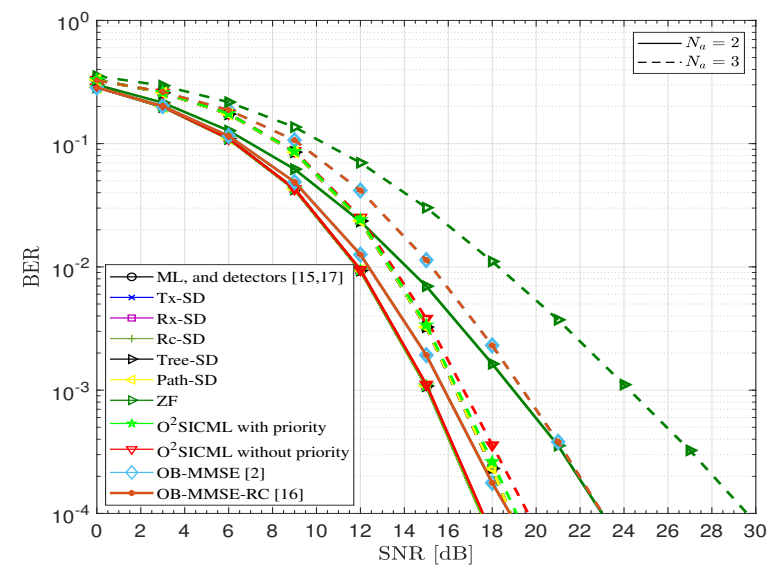

(a)

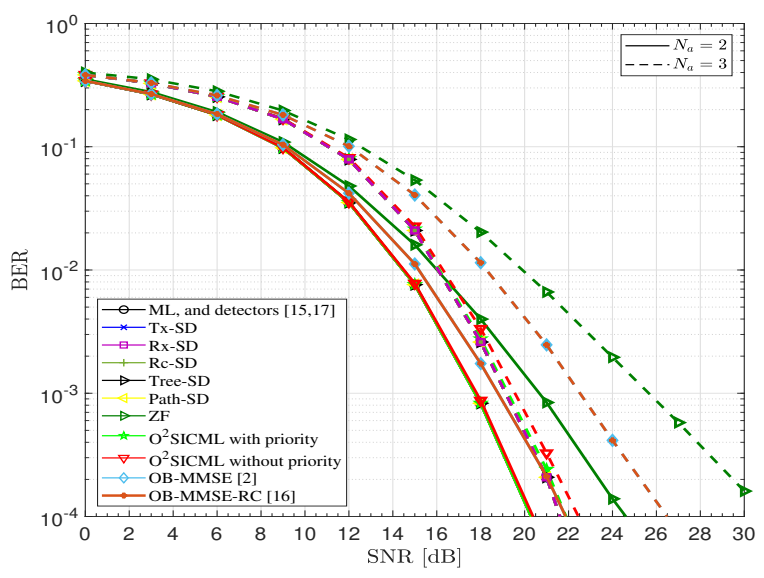

(b)

Fig. 4: GSM performance with $N_{t}=N_{r}=5, N_{a}=[2,3]$ and different linear and non-linear detectors: (a) 16QAM, (b) 32QAM.

priority. However, a gain of 1.7 (4) dB with 16QAM and 1.7 (4.7) dB with 32QAM are achieved compared to the lowcomplexity OB-MMSE when using $N_{a}=2\left(N_{a}=3\right)$. These results are maintained for large MIMO system, $N_{t}=8$ and $N_{a}=4$ with $M=[8,16]$ QAM as shown in Fig. 5 .

In addition, the relative complexity reduction (complexity saving) of $\mathrm{O}^{2} \mathrm{SIC}-\mathrm{ML}$ is compared to that of different SDbased detectors in Fig. 6. In addition, the complexity saving of the proposed detector is calculated using (15)-(16) for several configurations as shown in Table II. Although $\mathrm{O}^{2}$ SIC-ML, L-first-MMSE, and SA-SSDA achieve the optimal performance, but the upper-bound complexity saving of the proposed detector $(\bar{N}=N)$ is more significant in the order 99\%. Besides, OB-MMSE [2] and its reduced complexity version [16] show slightly better complexity saving but at the cost of significant performance degradation compared to $\mathrm{ML}$ as shown in Figs. 4-5. Thanks to the interference cancellation and the added ML verification step on a limited constellation subset ( $N_{e i}^{N_{a}}$ instead of $M^{N_{a}}$ with ML) that allowed $\mathrm{O}^{2}$ SIC- 
TABLE II: Complexity saving $C_{\text {Saving }}=1-\frac{C_{\text {detector }}}{C_{\text {ML }}}(\%)$ of the proposed detector compared to other low-complexity detectors.

\begin{tabular}{||c|c|c|c|c|c|c||}
\hline System Configuration & SE (bpcu) & O $^{2}$ SIC-ML $N_{e i}=2$ & OB-MMSE [2] & OB-MMSE-RC [16] & L-first-MMSE [15] & SA-SSDA [17] \\
\hline$N_{t}=8, N_{a}=2, N_{r}=8, M=16$ & 12 & 96.81 & 96.92 & 97.59 & 96.12 & 94.3 \\
\hline$N_{t}=8, N_{a}=2, N_{r}=8, M=64$ & 16 & 99.71 & 99.8 & 99.85 & 98.1 & 96.24 \\
\hline$N_{t}=32, N_{a}=6, N_{r}=6, M=16$ & 43 & 99.99 & 99.99 & 99.99 & 98.97 \\
\hline$N_{t}=32, N_{a}=6, N_{r}=6, M=64$ & 55 & 99.99 & 99.99 & 99.99 & 97.23 \\
\hline
\end{tabular}

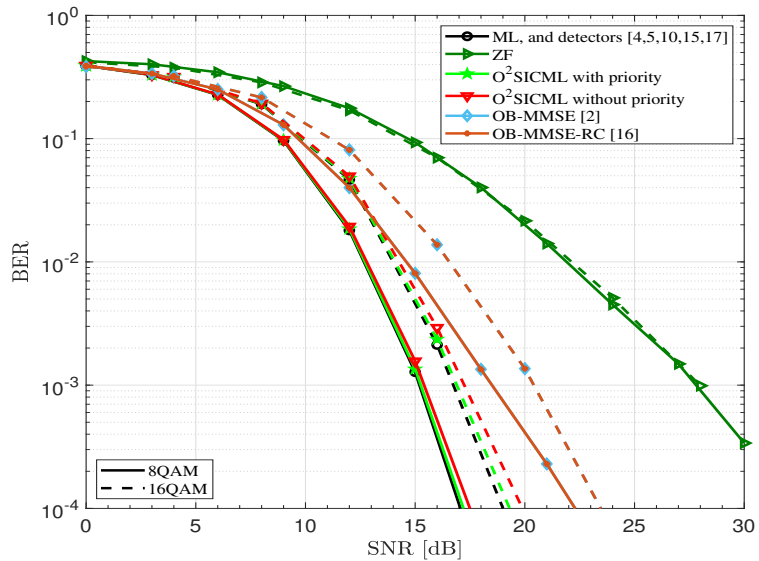

Fig. 5: GSM performance with $N_{t}=8, N_{r}=6, N_{a}=4, N_{e i}=2$ and $M=[8,16]$ QAM compared to linear and non-linear detectors.

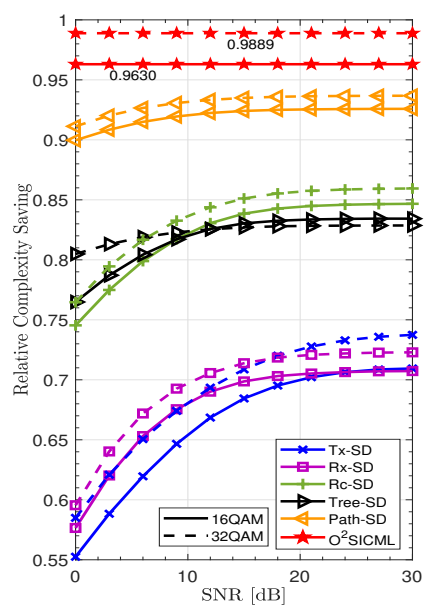

(a)

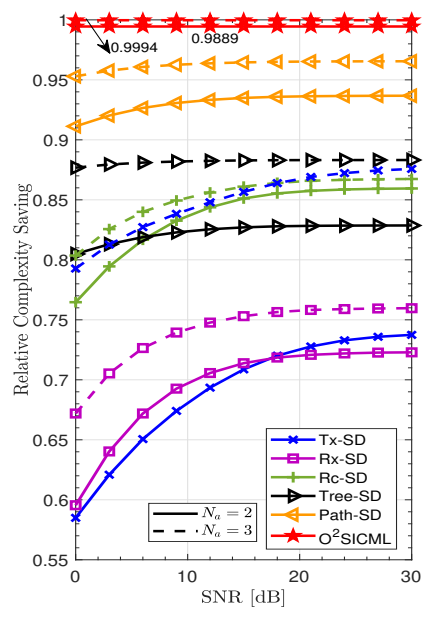

(b)
Fig. 6: Relative complexity reduction compared to ML of different optimal GSM detectors with $N_{t}=N_{r}=5$ : (a) $N_{a}=2$ and $M=$ [16, 32]QAM, (b) $N_{a}=[2,3]$ and 32QAM

ML to reduce the complexity prominently while attaining the optimal performance. Finally, we would like to highlight that the proposed detector reach the optimal performance even with $N_{r} \leq N_{t}$ as shown in Fig. 5 in contrast to the optimal SD based technique in [13] that can operate only when $N_{r} \geq N_{t} \geq N_{a}$. Note that the variable complexity of all SD-based techniques can be a drawback for the hardware implementation due to the variable detection delay compared to the fixed-rate proposed detector [14].

\section{CONCLUSION}

In this letter, we proposed $\mathrm{O}^{2} \mathrm{SIC}-\mathrm{ML}$ detector for GSM that generalizes many other schemes in the spatial IM domain, and it is a promising candidate for high data rates communications [12]. The proposed quasi-linear detector takes the advantages of ML and OSIC without their disadvantages of high complexity and possible error propagation, respectively.
More precisely, the $\mathrm{O}^{2}$ of the proposed algorithm comes from the: TAC ordering according to their reliability weight and ordering of APM symbol detection. The former ensures the low complexity by early search termination, while the latter allows a better interference cancellation. For further enhancement of OSIC performance, a set of $N_{e i}$ nearest neighbors for each raw symbol estimation is used to perform a treesearch and ML verification on a reduced subset. These adopted strategies give more control on the performance-complexity trade-off and allow reaching the optimal performance of nonlinear detectors with much lower complexity (dominant by $\bar{N} . N_{e i}^{N_{a}}$ where $\bar{N} \ll N$ and $\left.N_{e i} \ll M\right)$.

\section{REFERENCES}

[1] J. Wang, S. Jia and J. Song, "Generalised Spatial Modulation System with Multiple Active Transmit Antennas and Low Complexity Detection Scheme," IEEE Trans. Wireless Commun., vol. 11, no. 4, pp. 1605-1615, April 2012.

[2] Y. Xiao et al., "Low-Complexity Signal Detection for Generalized Spatial Modulation," IEEE Commun. Lett., vol. 18, no. 3, pp. 403-406, Mar. 2014.

[3] C. Chen et al., "An Improved Ordered-Block MMSE Detector for Generalized Spatial Modulation," IEEE Commun. Lett., vol. 19, no. 5, pp. 707-710, May 2015.

[4] A. Younis et al., "Generalised Sphere Decoding for Spatial Modulation," IEEE Trans. Commun., vol. 61, no. 7, pp. 2805-2815, July 2013.

[5] Y. Jiang et al., "Improved Low-Complexity Sphere Decoding for Generalized Spatial Modulation," IEEE Commun. Lett., vol. 22, no. 6, pp. 1164-1167, June 2018.

[6] A. Dhayni and H. Hijazi, "Low complexity maximum-likelihood-based method for estimating emitted symbols in a SM-MIMO receiver," STEricsson Invention Disclosure, U.S. Patent 20150030107, May 16, 2017.

[7] H. Hijazi, A. Haroun, M. Saad, A. C. Al Ghouwayel and A. Dhayni, "Near-Optimal Performance with Low-Complexity ML-Based Detector for MIMO Spatial Multiplexing," in IEEE Commun. Lett., vol. 25, no. 1, pp. 122-126, January 2021.

[8] Y. Jin Chun and S. Wu Kim, "Log-likelihood-ratio ordered successive interference cancellation in multi-user, multi-mode MIMO systems," IEEE Commun. Lett., vol. 12, no. 11, pp. 837 - 839, November 2008.

[9] M. Saad, A. Al-Ghouwayel and H. Hijazi, "UFMC Transceiver Complexity Reduction," IEEE 25th International Conf. on Telecom. (ICT), St. Malo, France, June 2018, pp. 295-301.

[10] I. Al-Nahhal, O. A. Dobre and S. S. Ikki, "Quadrature Spatial Modulation Decoding Complexity: Study and Reduction," IEEE Wireless Commun. Lett., vol. 6, no. 3, pp. 378-381, June 2017.

[11] Yeqiu Lan, "T-SD/P-SD", IEEE Dataport, 2017. [Online]. Available: http://dx.doi.org/10.21227/H2F93M. Accessed: Jun. 25, 2020.

[12] M. Saad, A. C. Al Ghouwayel, H. Hijazi, F. Bader and J. Palicot, "MIMO Techniques for Wireless Terabits Systems under Sub-THz Channel with RF Impairments," 2020 IEEE Int. Conf. on Commun. Workshops (ICC), Dublin, Ireland, June 2020, pp. 1-6.

[13] J. A. Cal-Braz and R. Sampaio-Neto, "Low-Complexity Sphere Decoding Detector for Generalized Spatial Modulation Systems," IEEE Commun. Lett., vol. 18, no. 6, pp. 949-952, June 2014.

[14] Z. Guo and P. Nilsson, "Algorithm and implementation of the K-best sphere decoding for MIMO detection," IEEE J. Sel. Areas Commun., vol. 24, no. 3, pp. 491-503, Mar. 2006.

[15] M. A. Simarro et al., "Low complexity Near-ML Sphere Decoding based on a MMSE ordering for Generalized Spatial Modulation," IEEE Int. Symp. on Personal, Indoor and Mobile Radio Commun., 2020, pp. 1-6.

[16] S. Wu, X. Tao and Y. Chen, "A New Constellation Diagram-Based Signal Detection for GSM-MIMO Systems," IEEE Commun. Let., vol. 22, no. 3, pp. 502-505, March 2018.

[17] T. Liu, C. Chen and C. Liu, "Fast Maximum Likelihood Detection of the Generalized Spatially Modulated Signals Using Successive Sphere Decoding Algorithms," IEEE Commun. Lett., vol. 23, no. 4, pp. 656659, April 2019. 